\title{
Avaliação de preditores de mortalidade intra-hospitalar em pacientes com Insuficiência Cardíaca em hospital terciário no Brasil
}

André Wajner ${ }^{1}$, Fernando Starosta de Waldemar ${ }^{1}$, Eduardo de Oliveira Fernandes $^{1}$, Janete Salles Brauner ${ }^{1}$, Guilherme Augusto Reissig Pereira $^{2}$, Tiago Landenberger ${ }^{2}$, Júlia De Luca Maccarini², Nicolas da Costa Peruzzo², Luis Eduardo Paim Rohde ${ }^{3}$

\section{Resumo}

Introdução: A Insuficiência Cardíaca (IC) representa a maior causa cardiovascular de morbidade hospitalar. Apesar da sua relevância, são escassos os estudos na América Latina sobre os preditores de mortalidade intrahospitalar em pacientes com IC.

Objetivo: Identificar os preditores de mortalidade intra-hospitalar em pacientes que internam por IC aguda ou que possuem IC e internam por outros motivos no Hospital Nossa Senhora da Conceição (HNSC) de Porto Alegre.

Materiais e Métodos: Coorte histórica de pacientes internados com diagnóstico de IC pelo Índice de Comorbidade de Charlson (ICCharlson) em 2009 e 2010.

Resultados: Foram avaliados 2056 pacientes que internaram no período de 01 de janeiro de 2009 a 31 de dezembro de 2010, sendo $51 \%$ homens e $49 \%$ mulheres. A idade média dos pacientes foi de 71 anos; a média do ICCharlson foi de 5 e IC aguda foi responsável por 28,7\% das internações. Encontramos na nossa amostra $361(17,4 \%)$ óbitos durante a internação, sendo 18,8\% no grupo de pacientes que internou por IC aguda e $16,8 \%$ no grupo com IC e que internou por outros motivos. Os principais preditores independentes de mortalidade intra-hospitalar no grupo que não internou por IC aguda foram: idade ( $\mathrm{RR}=1,023$; IC 95\% 1,011 - 1,036); tempo de permanência hospitalar 
(RR=1,009; IC 95\% 1,005 - 1,013); ICCharlson (RR=1,144; IC 95\% 1,098 1,193); doença renal ( $R R=2,49$; IC 95\% 1,71 - 3,63), demência ( $R R=1,94$; IC $95 \%$ 1,35 - 2,77) e doença neo-hematológica (RR=3,07; IC95\% 1,51-6,72). Já no grupo que internou por IC aguda os principais preditores independentes de mortalidade foram: idade ( $\mathrm{RR}=1,028$; IC 95\% 1,005 - 1,052); ICCharlson $(\mathrm{RR}=1,225$; IC 95\% 1,137 - 1,320); doença renal $(\mathrm{RR}=2,971$; IC 95\% 1,587 5,560); e demência ( $R R=2,179$ IC 95\% 1,099 - 4,318).

Conclusão: Nosso trabalho mostrou um perfil de pacientes com IC diferente daqueles estudados na América do Norte e Europa. É um dos poucos estudos existentes a ressaltar a importância do ICCharlson em predizer mortalidade em pacientes com IC e a analisar as características de uma população portadora de IC que interna por outros motivos que não a IC aguda. Além disso, constatamos que, apesar de termos pacientes com características diferentes da literatura internacional, a maioria dos preditores de mortalidade intra-hospitalar encontrados foram muito semelhantes aos dos estudos já publicados e também foram muito semelhantes entre as duas populações analisadas pelo nosso trabalho.

Palavras Chave: Mortalidade intra-hospitalar, insuficiência cardíaca, doença cardiovascular.

1: Médicos orientadores do TCC; awajner@gmail.com; fernandowaldemar@gmail.com;

2: Médicos residentes do serviço de Clínica Médica do HNSC;

3: Professor orientador 


\section{INTRODUÇÃO}

A Insuficiência Cardíaca (IC) é um problema de saúde comum, associado a morbidade e mortalidade consideráveis. Apesar do declínio da prevalência de inúmeras outras doenças cardíacas ${ }^{1}$, a prevalência da IC, em razão de seu caráter crônico, cresce à medida que aumenta a expectativa de vida da população; e que surgem abordagens terapêuticas que aumentam a sobrevida de pacientes com doenças cardiovasculares ${ }^{2}$. Dados atualizados da American Heart Association estimam uma prevalência de IC de 5,1 milhões de indivíduos somente nos EUA, entre 2007 e 2012, com projeções de maior aumento até $2030^{3}$.

Embora tenha havido progresso no tratamento da IC, a doença permanece como uma das principais causas de hospitalização em vários países e está associada com elevadas taxas de morbidade, mortalidade e custos para a saúde ${ }^{4}$. Trata-se da principal causa de hospitalização em pacientes acima de 65 anos nos $\mathrm{EUA}^{5}$, bem como a principal causa de hospitalização na América do Sul, com base em dados disponíveis sobre cerca de 50\% da população ${ }^{6}$. No Brasil, segundo dados disponibilizados pelo DATA-SUS, entre todas as causas de admissão hospitalar por doenças circulatórias, em 2012, IC foi responsável por $21 \%{ }^{7}$. Quanto à mortalidade intra-hospitalar de pacientes com IC, dados disponibilizados pelo DATA-SUS apontam para uma taxa de 9,6\%, semelhante àquela do Registro BREATHE (coorte multicêntrica brasileira sobre IC), que foi de $12 \%^{8}$. Devido à relevância da IC, entidades médicas instituíram protocolos que servem como marcadores de qualidade no atendimento dessa doença por uma instituição ${ }^{9}$; tais protocolos incluem a avaliação da função sistólica do ventrículo esquerdo, a prescrição de medicamentos que alteram sobrevida e orientações sobre hábitos de vida no momento da alta ${ }^{9}$.

Ainda no contexto de internação hospitalar de portadores de IC, um tópico relevante, embora ainda pouco estudado, é a análise de características e de marcadores prognósticos de pacientes internados por outras causas ${ }^{10,11}$. Pressupõe-se que esse grupo de pacientes, muito provavelmente, beneficiar-se-ia das mesmas condutas implementadas para os indivíduos com IC aguda.

Em estudo americano recente, foram comparadas as taxas de aderência a medidas de cuidado para IC em ambiente hospitalar entre os pacientes que internaram por IC aguda e aqueles que internaram por outras causas ${ }^{12}$. Os resultados dessa coorte revelaram que 
indivíduos hospitalizados por IC aguda tiveram maior proporção de aferição de função ventricular esquerda em comparação com os indivíduos internados por outras causas. A mesma tendência foi observada na prescrição de inibidores da ECA (iECA) ou bloqueadores do receptor de angiotensina (BRA) na alta; e na de antagonistas da aldosterona. Já em relação a betabloqueadores, não se observou diferença entre os grupos. Destaca-se, ainda, que a taxa de mortalidade em um ano foi semelhante entre os dois grupos estudados. Em ambos os grupos houve redução da mortalidade nos que tiveram o ventrículo esquerdo avaliado, e o mesmo foi observado naqueles que receberam prescrição de iECA/BRA e betabloqueadores na alta. O uso de antagonistas da aldosterona em indivíduos com FE reduzida foi associado com redução de mortalidade pós-hospitalar, mas sem significância estatística. Quanto à anticoagulação de pacientes com fibrilação atrial, houve redução na mortalidade entre os indivíduos hospitalizados por outras causas, fato não observado entre aqueles internados por IC aguda.

Outro estudo norte-americano, uma subanálise do CHARM Program, avaliou mortalidade em pacientes portadores de IC com internação prévia por IC aguda ${ }^{13}$. Após um seguimento mediano de 37,7 meses, tais pacientes foram divididos em três grupos: pacientes que não voltaram a ser hospitalizados $(37,1 \%)$; pacientes que foram hospitalizados por causas cardiovasculares $(38,1 \%)$; e pacientes que foram hospitalizados por causas nãocardiovasculares $(24,9 \%)$. As taxas de mortalidade dos grupos de pacientes hospitalizados por causas cardiovasculares e de pacientes hospitalizados por causas não-cardiovasculares foram maiores do que a do grupo de pacientes que não voltaram a ser hospitalizados (riscos relativos de 6,3 e 5,8, respectivamente), e tais resultados tiveram significância estatística. $\mathrm{Na}$ análise feita entre os grupos de pacientes que voltaram a ser hospitalizados (e que sobreviveram a essa internação), a mortalidade em 30 dias após a reinternação foi maior no grupo de pacientes hospitalizados por causas cardiovasculares do que no grupo de pacientes hospitalizados por causas não-cardiovasculares (90,4 versus 55.9 por 100 pacientes-anos; $\mathrm{p}<0,001)$. Entretanto, não houve diferença estatisticamente significativa entre as taxas de mortalidade de ambos os grupos, a longo prazo (14,5 versus 14,6 por 100 pacientes-anos; $\mathrm{p}=0,85)$.

Outro estudo, esse conduzido em Israel, avaliou pacientes portadores de IC internados por causas secundárias ${ }^{14}$. Tais pacientes foram hospitalizados, em sua maioria, por infarto 
agudo do miocárdio, insuficiência renal aguda, anemia, exacerbação de doença pulmonar obstrutiva crônica ou pneumonia; e, em comparação com pacientes internados primariamente pela IC, eram mais jovens, tinham menos comorbidades (exceto por doença arterial coronariana), a duração de sua internação foi mais longa, e a mortalidade intra-hospitalar, mais elevada. Por outro lado, ao se analisar a mortalidade em 10 anos, observou-se que ela foi maior entre pacientes cuja causa primária da internação foi a própria IC.

Um estudo de coorte norueguês recentemente publicado avaliou o desenvolvimento de IC em pacientes internados por infarto agudo do miocárdio ${ }^{15}$. Entre os pacientes que apresentaram IC já na admissão hospitalar ou durante a internação, foram preditores do desenvolvimento de IC: idade mais avançada, sexo masculino, diabete melito, doença pulmonar obstrutiva crônica e fibrilação atrial. Eventos cardíacos adversos (como fibrilação ventricular e bloqueio atrioventricular) durante a internação foram mais comuns nesses pacientes do que entre aqueles que não se mostraram portadores de IC na admissão hospitalar ou durante a internação. Quanto aos pacientes que tiveram alta hospitalar sem diagnóstico de IC, $12,6 \%$ foram internados ou faleceram por IC em um tempo mediano de seguimento de 3,2 anos; foram preditores para o desenvolvimento de IC, nesse grupo de pacientes, idade mais avançada, maior tempo de hospitalização-índice, diabete melito, doença pulmonar obstrutiva crônica, insuficiência renal e fibrilação atrial.

Quanto à IC no cenário brasileiro, apesar da grande relevância da doença no nosso país, a literatura ainda carece de estudos locorregionais ou mesmo nacionais sobre o assunto. A maioria das pesquisas existentes sobre IC foi realizada nos Estados Unidos ou na Europa e reuniu pacientes com IC com fração de ejeção (FE) reduzida, mais saudáveis que os pacientes atendidos no sistema público de saúde brasileiro e, provavelmente, com um perfil socioeconômico diferente daquele dos nossos pacientes ${ }^{10}$. Mesmo na América Latina como um todo, há escassez de estudos sobre os indicadores demográficos, hospitalares e assistenciais nessa população ${ }^{16}$.

Em vista do exposto, obter mais indicadores prognósticos de pacientes com IC, tais como preditores de morbimortalidade intra-hospitalar, no cenário da saúde pública brasileira, é de grande valia, já que pode auxiliar na estratificação desses pacientes e na otimização dos cuidados a eles prestados ${ }^{17}$ Nesse sentido, é possível que informações simples, tais como o 
real motivo da internação do paciente portador de IC crônica (se por IC aguda ou outra causa), tenham grande valor prognóstico.

\section{OBJETIVO}

O objetivo do presente estudo é identificar os preditores de mortalidade intrahospitalar em pacientes que internam por IC aguda ou que possuem IC e internam por outros motivos, no Hospital Nossa Senhora da Conceição (HNSC) de Porto Alegre.

\section{MATERIAIS E MÉTODOS}

\subsection{Local, Delineamento e Pacientes}

Este estudo foi realizado no Hospital Nossa Senhora da Conceição (HNSC) de Porto Alegre, Rio Grande do Sul, Brasil, um hospital público de nível terciário, com aproximadamente 850 leitos. Trata-se de estudo de coorte prospectivo, em que foram incluídos pacientes adultos $(\geq$ 18 anos) que internaram em qualquer enfermaria ou Unidade de Tratamento Intensivo (UTI) deste hospital e tenham sido identificados como portadores de IC pelo médico assistente no Índice de Comorbidade de Charlson (ICCharlson), via prontuário eletrônico. Foram excluídos da análise pacientes pediátricos (idade < 18 anos), com permanência apenas no setor de emergência (sem internar na enfermaria ou na UTI), com evasão hospitalar e com nota de alta informatizada indisponível.

Na nossa instituição, o ICCharlson é preenchido pelo médico assistente no prontuário eletrônico de forma compulsória, no momento da internação e na alta hospitalar. A falta de preenchimento impede a continuidade de procedimentos diagnósticos e terapêuticos, ou a alta hospitalar. O ECCh é uma excelente ferramenta de uso hospitalar para predição clínica de mortalidade intra-hospitalar em uma população geral ${ }^{18}$. Ele é composto por um escore de várias comorbidades e é amplamente utilizado para classificar a gravidade dos pacientes, sendo possível comparar a carga de doenças de pacientes de diferentes serviços médicos e hospitalares. O ICCharlson é composto por: infarto do miocárdio (IAM), insuficiência cardíaca congestiva, doença vascular periférica e da aorta, doença cerebrovascular, demência, doença pulmonar obstrutiva crônica (DPOC), doença do tecido conjuntivo, doença ulcerosa, hepatopatia, diabetes, doença renal moderada a grave, hemiplegia, linfoma/mieloma, leucemia/policitemia vera, tumor, AIDS e câncer metastático ${ }^{18}$. 


\subsection{Logística e Coleta de Dados}

Para fins de análise, pacientes que apresentaram múltiplas internações tiveram considerada apenas a sua última hospitalização, para que fossem englobados todos os óbitos intrahospitalares da amostra, evitando que pacientes mais graves e com múltiplas reinternações tivessem suas características analisadas múltiplas vezes e buscando preservar a independência dos dados. A coleta de dados foi realizada por médicos residentes de clínica médica, previamente treinados, através da revisão sistemática de prontuários eletrônicos. Houve a verificação de $10 \%$ da amostra por outros dois pesquisadores do estudo, médicos preceptores do serviço de Medicina Interna, para aferição da confiabilidade dos dados coletados. Foi criado um protocolo de coleta informatizado e totalmente integrado ao prontuário eletrônico do Hospital. Os pacientes foram selecionados através de um sistema informatizado com identificação automática de todos aqueles que preenchiam os critérios de inclusão.

Foram incluídos na coleta de dados as seguintes variáveis e instrumentos: idade; sexo; raça; procedência (Porto Alegre, Região Metropolitana de Porto Alegre e Interior); equipe em que o paciente esteve internado (Cardiologia, Medicina Interna e Outras); tempo de permanência hospitalar; causa da internação; ICCharlsonarlson; valores laboratoriais (uréia, sódio, creatinina e potássio) nas primeiras 24 horas de hospitalização; dados ecocardiográficos até um ano antes da alta hospitalar: fração de ejeção do ventrículo esquerdo (FEVE), hipertrofia ventricular esquerda, presença de hipocinesia difusa ou alterações segmentares de contratilidade e lesões valvares; internação em UTI; e óbito intrahospitalar.

A amostra foi separada em dois grupos: pacientes que tinham IC, mas que internaram por outro motivo que não IC aguda descompensada (grupo IC não-aguda); e pacientes que internaram por IC aguda descompensada (grupo IC aguda). Esse último grupo era definido quando o paciente apresentasse no diagnóstico principal, preenchido pelo médico assistente no momento da alta hospitalar, um dos seguintes Códigos de Doença Internacional (CID): I11.0- Doença cardíaca hipertensiva com insuficiência cardíaca (congestiva); I13.0- Doença cardíaca e renal hipertensiva com insuficiência cardíaca (congestiva); I13.2- Doença cardíaca e renal hipertensiva com insuficiência cardíaca (congestiva) e insuficiência renal; I42.0Cardiomiopatia dilatada; I42.6- Cardiomiopatia alcoólica; I42.8- Outras cardiomiopatias; 
I42.9- Cardiomiopatia não especificada; I50.0- Insuficiência Cardíaca Congestiva; I50.1Insuficiência ventricular esquerda; I.50.9- Insuficiência cardíaca não especificada; e J.81Edema pulmonar, não especificado de outra forma.

\subsection{Análise dos dados}

Variáveis contínuas foram expressas como média e desvio-padrão ou mediana e intervalo interquartil (IIQ 25\%-75\%). Variáveis categóricas foram expressas como frequência e percentuais. Análises univariadas foram realizadas pelo teste T de Student, teste de Poisson e teste de Qui-quadrado. Para as análises multivariadas foi realizada a Regressão de Poisson com estimativa de variâncias robustas, calculando-se as razões de incidência e os intervalos de confiança de $95 \%$. A partir dos dados coletados dos pacientes foram realizadas análises univariadas de variáveis contínuas e categóricas dentro de cada um dos dois grupos prédefinidos (pacientes que internam por IC aguda e pacientes que internam por outras causas). As variáveis que na análise univariada apresentaram um valor de $\mathrm{p}<0,20$ foram selecionadas para a análise multivariada, para identificação de preditores de mortalidade intra-hospitalar. Um valor de p bicaudal de 5\% foi considerado estatisticamente significativo. Os dados coletados no sistema informatizado customizado para a pesquisa foram exportados para uma planilha de Microsoft Excel (versão 18), e as análises estatísticas foram conduzidas pelo SPSS Basic (versão 19.0).

O projeto de pesquisa foi aprovado no Comitê de Ética em Pesquisa do GHC (Grupo Hospitalar Conceição). Os participantes envolvidos no estudo não foram submetidos a nenhum grau de risco, pois esse trabalho é observacional, retrospectivo e com análise de dados secundários. Por este motivo, o termo de consentimento livre e esclarecido não foi considerado necessário pelo Comitê de Ética. Houve total sigilo de dados pessoais, e as informações foram coletadas exclusivamente para fins de pesquisa. Não houve fontes de financiamento do estudo.

\section{RESULTADOS}

Foram avaliados 2.056 pacientes que internaram no período de $1^{\circ}$ de janeiro de 2009 a 31 de dezembro de 2010, de acordo com os critérios de inclusão e exclusão descritos acima. As características dos pacientes da amostra estão listadas na Tabela 1. 
Tabela 1: Características basais dos pacientes. ( ${ }^{*}$ Quando Creatinina $\geq 3,0$. ${ }^{* *}$ Valores laboratoriais nas primeiras 24 horas da admissão).

\begin{tabular}{|c|c|c|c|c|}
\hline & $\begin{array}{l}\text { Todos } \\
(n=2.056)\end{array}$ & $\begin{array}{l}\text { Internados por IC } \\
\text { aguda }(n=590)\end{array}$ & $\begin{array}{l}\text { Internados por outra } \\
\text { causa }(n=1466)\end{array}$ & Valor $p$ \\
\hline Idade & 71 (61-79) & $70(60-79)$ & $71(61-80)$ & 0,091 \\
\hline Sexo masculino & $1041(51 \%)$ & 301 (51\%) & 740 (50\%) & 0,84 \\
\hline Raça branca & $1736(84 \%)$ & $488(83 \%)$ & $1248(85 \%)$ & 0,179 \\
\hline $\begin{array}{l}\text { Tempo de } \\
\text { permanência }\end{array}$ & $15(10-23)$ & $13(9-20)$ & $16(10-24)$ & $<0,001$ \\
\hline Fração de Ejeção & $44(33-62)$ & $38(30-50)$ & $48(37-64)$ & $<0,001$ \\
\hline Escore Charlson & $5(4-7)$ & $5,0(4-7)$ & $6,0(4-7)$ & $<0,001$ \\
\hline Passagem pela UTI & $362(18 \%)$ & $87(15 \%)$ & 275 (19\%) & 0,035 \\
\hline $\begin{array}{l}\text { Doença } \\
\text { cerebrovascular }\end{array}$ & $361(18 \%)$ & 65 (11\%) & $296(20 \%)$ & $<0,001$ \\
\hline IAM & $504(24 \%)$ & $115(20 \%)$ & $389(26 \%)$ & 0,001 \\
\hline Diabetes Mellitus & 647 (31\%) & $171(29 \%)$ & $476(32 \%)$ & 0,128 \\
\hline Doença Renal* & 287 (14\%) & $81(14 \%)$ & $206(14 \%)$ & 0,88 \\
\hline $\begin{array}{l}\text { Doença Vascular } \\
\text { Periférica }\end{array}$ & 307 (15\%) & $59(10 \%)$ & $248(17 \%)$ & $<0,001$ \\
\hline Neoplasia & $121(6 \%)$ & $16(3 \%)$ & $105(7 \%)$ & $<0,001$ \\
\hline DPOC & $472(23 \%)$ & $106(18 \%)$ & $366(25 \%)$ & 0,001 \\
\hline Demência & $174(8 \%)$ & $41(7 \%)$ & $133(9 \%)$ & 0,136 \\
\hline Doença Hepática & $86(4 \%)$ & $29(5 \%)$ & $57(4 \%)$ & 0,33 \\
\hline Uréia** & $56(41-83)$ & $55(41-83)$ & $56(40-84)$ & 0,760 \\
\hline Creatinina** & $1,21(0,92-1,66)$ & $1,2(0,96-1,60)$ & $1,21(0,9-1,69)$ & 0,503 \\
\hline Sódio** & $138(136-141)$ & $139(136-141)$ & $138(136-141)$ & 0,007 \\
\hline
\end{tabular}


Potássio**

Óbitos
$4,4(4,0-4,8)$

$361(17,4 \%)$
$4,3(4,0-4,8)$

$112(18,8 \%)$
$4,4(4,0-4,8)$

0,125

Fonte: criação própria do estudo.

A distribuição entre os sexos foi homogênea (51\% de homens, 49\% de mulheres); a idade média dos pacientes foi de 71 anos; a maioria dos pacientes era procedente de Porto Alegre $(59,3 \%)$ e internou principalmente nas equipes de Cardiologia (37,8\%) e Medicina Interna (29\%). A mediana do tempo de permanência no hospital foi de 15 dias (IIQ 25-75\%: 10-23). A fração de ejeção média dos pacientes foi de $44 \%$, e mostrou-se significativamente menor no grupo de pacientes IC aguda (38\%); a média do ICCharlson foi de 5 na população geral do estudo, e mostrou-se significativamente maior no grupo IC não-aguda (6); IC aguda foi responsável por $28,7 \%$ das internações.

Na nossa amostra, 361 pacientes $(17,4 \%)$ foram a óbito durante a internação, o que correspondeu a $18,8 \%$ de mortalidade no grupo de pacientes com IC aguda e a 16,8\% de mortalidade no grupo de pacientes com IC não-aguda.

Foram selecionadas para a análise multivariada apenas as variáveis com valor de p<0,20 na análise univariada (Tabelas 2 e 3). As variáveis significativas na análise univariada, tanto para o grupo IC aguda quanto para o grupo IC não-aguda, foram: idade; tempo de permanência; ICCharlson; potássio; uréia; procedência; neoplasia sólida; doença neo-hematológica; demência; doença cerebrovascular; doença renal; doença vascular periférica e da aorta; e doença hepática.

Fração de ejeção $\leq 40 \%$ e insuficiência tricúspide grave foram significativas na análise univariada apenas para o grupo IC não-aguda. Já o valor de sódio nas primeiras 24h, creatinina, DPOC e IAM foram significativas apenas para o grupo IC aguda.

Tabela 2: Análise univariada dos preditores de mortalidade no grupo IC não-aguda.

\begin{tabular}{|lll|}
\hline Variáveis Contínuas Preditoras & RR (IC 95\%) & Valor de $\mathbf{p}$ \\
\hline Idade & $1,028(1,018-1,038)$ & $<0,0001$ \\
\hline Tempo de permanência & $1,007(1,003-1,011)$ & $<0,0001$ \\
\hline
\end{tabular}




\begin{tabular}{|c|c|c|}
\hline ICCharlson & $1,182(1,137-1,229)$ & $<0,0001$ \\
\hline Potássio & $1,206(1,040-1,399)$ & 0,008 \\
\hline Uréia & $1,004(1,002-1,006)$ & $<0,0001$ \\
\hline Variáveis Categóricas Preditoras & Óbitos & Valor de $p$ \\
\hline $\begin{array}{l}\text { Procedência } \\
\text { Porto Alegre } \\
\text { Região Metropolitana de POA } \\
\text { Interior }\end{array}$ & $\begin{array}{l}157(18,4 \%) \\
75(14,6 \%) \\
17(15,3 \%)\end{array}$ & 0,1 \\
\hline $\begin{array}{l}\text { FE } \leq 40 \% \\
\text { Não } \\
\text { Sim }\end{array}$ & $\begin{array}{l}74(18,8 \%) \\
113(14,8 \%)\end{array}$ & 0,186 \\
\hline $\begin{array}{l}\text { Insuficiência Tricúspide Grave } \\
\text { Não } \\
\text { Sim }\end{array}$ & $\begin{array}{l}190(16,1 \%) \\
5(29,4 \%)\end{array}$ & 0,147 \\
\hline $\begin{array}{l}\text { Neoplasia Sólida } \\
\text { Não } \\
\text { Sim }\end{array}$ & $\begin{array}{l}220(16,1 \%) \\
29(27,6 \%)\end{array}$ & 0,007 \\
\hline $\begin{array}{l}\text { Doença Neo-Hematológica } \\
\text { Não } \\
\text { Sim }\end{array}$ & $\begin{array}{l}243(16,7 \%) \\
6(35,3 \%)\end{array}$ & 0,072 \\
\hline $\begin{array}{l}\text { Demência } \\
\text { Não } \\
\text { Sim }\end{array}$ & $\begin{array}{l}201(15 \%) \\
48(36,1 \%)\end{array}$ & $<0,0001$ \\
\hline $\begin{array}{l}\text { Doença Cerebrovascular } \\
\text { Não } \\
\text { Sim }\end{array}$ & $\begin{array}{l}171(14,5 \%) \\
78(26,4 \%)\end{array}$ & $<0,0001$ \\
\hline $\begin{array}{l}\text { Doença Renal } \\
\text { Não } \\
\text { Sim }\end{array}$ & $\begin{array}{l}175(13,8 \%) \\
74(35,9 \%)\end{array}$ & $<0,0001$ \\
\hline $\begin{array}{l}\text { Doença Vascular Periférica e da Aorta } \\
\text { Não } \\
\text { Sim }\end{array}$ & $\begin{array}{l}199(16,2 \%) \\
50(20,2 \%)\end{array}$ & 0,184 \\
\hline $\begin{array}{l}\text { Doença Hepática } \\
\text { Não } \\
\text { Sim }\end{array}$ & $\begin{array}{l}235(16,6 \%) \\
14(24,6 \%)\end{array}$ & 0,159 \\
\hline
\end{tabular}

Fonte: criação própria do estudo. 
Tabela 3: Análise univariada dos preditores de mortalidade no grupo IC aguda.

\begin{tabular}{|c|c|c|}
\hline Variáveis Contínuas Preditoras & RR (IC 95\%) & Valor de $p$ \\
\hline Idade & $1,025(1,010-1,040)$ & 0,001 \\
\hline Tempo de permanência & $1,006(1,003-1,010)$ & 0,001 \\
\hline ICCharlson & $1,285(1,206-1,368)$ & $<0,0001$ \\
\hline Potássio & $1,481(1,201-1,826)$ & $<0,0001$ \\
\hline Uréia & $1,010(1,007-1,013)$ & $<0,0001$ \\
\hline Creatinina & $1,169(1,031-1,326)$ & 0,015 \\
\hline Sódio & $0,954(0,917-0,993)$ & 0,022 \\
\hline Variáveis Categóricas Preditoras & Óbitos & Valor de $p$ \\
\hline $\begin{array}{l}\text { Procedência } \\
\text { Porto Alegre } \\
\text { Região Metropolitana de POA } \\
\text { Interior }\end{array}$ & $\begin{array}{l}81(21,5 \%) \\
27(14,4 \%) \\
4(12,5 \%)\end{array}$ & 0,074 \\
\hline $\begin{array}{l}\text { Neoplasia Sólida } \\
\text { Não } \\
\text { Sim }\end{array}$ & $\begin{array}{l}105(18,1 \%) \\
7(43,8 \%)\end{array}$ & 0,025 \\
\hline $\begin{array}{l}\text { Doença Neo-Hematológica } \\
\text { Não } \\
\text { Sim }\end{array}$ & $\begin{array}{l}110(18,6 \%) \\
2(66,7 \%)\end{array}$ & 0,075 \\
\hline $\begin{array}{l}\text { Demência } \\
\text { Não } \\
\text { Sim }\end{array}$ & $\begin{array}{l}93(16,8 \%) \\
19(46,3 \%)\end{array}$ & $<0,0001$ \\
\hline $\begin{array}{l}\text { Doença Cerebrovascular } \\
\text { Não } \\
\text { Sim }\end{array}$ & $\begin{array}{l}86(16,3 \%) \\
26(38,8)\end{array}$ & $<0,0001$ \\
\hline $\begin{array}{l}\text { DPOC } \\
\text { Não } \\
\text { Sim }\end{array}$ & $\begin{array}{l}84(17,2 \%) \\
28(25,9 \%)\end{array}$ & 0,054 \\
\hline $\begin{array}{l}\text { Doença Renal } \\
\text { Não } \\
\text { Sim }\end{array}$ & $\begin{array}{l}70(13,6 \%) \\
42(51,2 \%)\end{array}$ & $<0,0001$ \\
\hline $\begin{array}{l}\text { Doença Vascular Periférica e da Aorta } \\
\text { Não } \\
\text { Sim }\end{array}$ & $\begin{array}{l}89(16,6 \%) \\
23(39 \%)\end{array}$ & $<0,0001$ \\
\hline
\end{tabular}




\begin{tabular}{|c|c|c|}
\hline Doença Hepática & & 0,131 \\
\hline Não & $103(18,2 \%)$ & \\
\hline Sim & $9(30 \%)$ & \\
\hline IAM & & 0,002 \\
\hline Não & $77(16,1 \%)$ & \\
\hline Sim & $35(30,2 \%)$ & \\
\hline
\end{tabular}

Fonte: criação própria do estudo.

Na análise multivariada do grupo IC não-aguda (Tabela 4), o tempo de permanência hospitalar associou-se de forma independente a um maior risco de óbito intra-hospitalar $(\mathrm{RR}=1,009 ; \mathrm{IC} 95 \%$ 1,005 - 1,013).

Variáveis como idade, doença renal, demência e doença neo-hematológica foram analisadas sem a presença do ICCharlson, para evitar um efeito de multicolinearidade, e também apresentaram significância estatística: idade ( $R R=1,023$; IC 95\% 1,011 - 1,036); doença renal ( $R R=2,49$; IC 95\% 1,71 - 3,63); demência ( $R R=1,92$; IC 95\% 1,35 - 2,77) e doença neo-hematológica ( $R R=3,07$; IC 95\% 1,51 - 6,72). Da mesma forma, o ICCharlson apresentou multicolinearidade com as comorbidades que o compõem. Logo, no momento de sua análise, foram retiradas as comorbidades que fazem parte da sua composição, apresentando com isso significância estatística (RR=1,144; IC 95\% 1,098 - 1,193).

A presença de $\mathrm{FE} \leq 40 \%$ no Ecocardiograma não foi associada à mortalidade intrahospitalar de forma significativa ( $R R=0,99 ;$ IC $95 \% 0,98-1,00)$.

Na análise multivariada do grupo IC não-aguda (Tabela 4), o tempod e permanência hospitalar associou-se de forma independente a um maior risco de óbito intra-hospitalar (RR=1,009; IC 95\% 1,005 - 1,013).

Variáveis como idade, doença renal, demência e doença neo-hematológica foram analisadas sem a presença do ICCharlson, para evitar um efeito de multicolinearidade, e também apresentaram significância estatística: idade ( $R R=1,023$; IC 95\% 1,011 - 1,036); doença renal $(\mathrm{RR}=2,49$; IC 95\% 1,71 - 3,63); demência $(\mathrm{RR}=1,92$; IC 95\% 1,35 - 2,77) e doença neo-hematológica ( $R R=3,07$; IC 95\% 1,51 - 6,72). Da mesma forma, o ICCharlson apresentou multicolinearidade com as comorbidades que o compõem. Logo, no momento de 
sua análise, foram retiradas as comorbidades que fazem parte da sua composição, apresentando com isso significância estatística ( $R R=1,144$; IC 95\% 1,098 - 1,193).

A análise $\mathrm{FE} \leq 40 \%$ no Ecocardiograma não foi significativa ( $\mathrm{RR}=0,99$; IC 95\% 0,98 $-1,00)$.

Tabela 4: Análise multivariada dos preditores de mortalidade no grupo IC não-aguda ( ${ }^{*}$ Resultado após a retirada de neoplasia sólida, doença cerebrovascular, doença renal, doença vascular periférica e da aorta, demência e idade, devido a efeito de multicolinearidade com ICCharlson. ${ }^{* *}$ Resultado após a retirada do ICCharlson, devido a efeito de multicolinearidade com as comorbidades acima).

\begin{tabular}{|c|c|c|c|c|}
\hline Preditores & $\begin{array}{c}\text { RR }(\text { IC 95\%) } \\
\text { *Com Charlson }\end{array}$ & $\begin{array}{l}\text { Valor de p } \\
\text { *Com } \\
\text { Charlson }\end{array}$ & $\begin{array}{c}\text { RR (IC 95\%) } \\
* * \text { Sem Charlson }\end{array}$ & $\begin{array}{c}\text { Valor de p } \\
\text { ***:Sem } \\
\text { Charlson }\end{array}$ \\
\hline Idade $* *$ & NA & NA & $\begin{array}{c}1,023 \\
(1,011-1,036)\end{array}$ & $<0,0001$ \\
\hline Tempo de permanência & $\begin{array}{c}1,009 \\
(1,005-1,013)\end{array}$ & $<0,0001$ & $\begin{array}{c}1,01 \\
(1,00-1,01)\end{array}$ & $<0,0001$ \\
\hline ICCharlson* & $\begin{array}{c}1,144 \\
(1,098-1,193)\end{array}$ & $<0,0001$ & NA & NA \\
\hline Doença Renal & NA & NA & $2,49(1,71-3,63)$ & $<0,0001$ \\
\hline Demência & NA & NA & $1,92(1,35-2,77)$ & $<0,0001$ \\
\hline Doença Neo-Hematológica & NA & NA & $3,07(1,51-6,72)$ & 0,002 \\
\hline $\begin{array}{l}\mathrm{FE} \leq 4 \mathbf{4 0 \%} \text { no } \\
\text { Ecocardiograma }\end{array}$ & $0,99(0,98-1,00)$ & 0,039 & $0,98(0,97-0,99)$ & 0,055 \\
\hline
\end{tabular}

Fonte: criação própria do estudo.

Na análise multivariada do grupo IC aguda (Tabela 5), a insuficiência tricúspide grave foi associada de forma independente a um maior risco de óbito intra-hospitalar $(\mathrm{RR}=2,90$ IC 95\% 1,24 - 6,81). Na análise das variáveis idade, doença renal, demência, doença neo- 
hematológica e IAM sem a presença do ICCharlson, obtiveram-se os seguintes resultados: idade ( $R R=1,03$; IC 95\% 1,01 - 1,05); doença renal ( $R R=2,97$; IC 95\% 1,59 - 5,56); demência $(\mathrm{RR}=2,18$; IC 95\% 1,09 - 4,32); doença neo-hematológica $(\mathrm{RR}=4,083$; IC 95\% 0,973 17,140); IAM (RR=1,46; IC 95\% 0,88 - 2,41). O ICCharlson também foi analisado neste grupo após a retirada da análise das comorbidades que o compõe e confirmou ser um importante preditor de mortalidade ( $R R=1,225$; IC 95\% 1,137 - 1,320).

Tabela 5: Análise multivariada dos preditores de mortalidade no grupo IC aguda ( ${ }^{*}$ Resultado após a retirada de neoplasia sólida, doença cerebrovascular, doença renal, doença vascular periférica e da aorta, demência, doença hepática, doença neo-hematológica, doença pulmonar, IAM e idade, por efeito de multicolinearidade com ICCharlson. ** Resultado após a retirada do ICCharlson, devido a efeito de multicolinearidade com as comorbidades acima).

\begin{tabular}{|lcccc|}
\hline Preditores & $\begin{array}{c}\text { RR (IC 95\%) } \\
\text { *Com Charlson }\end{array}$ & $\begin{array}{c}\text { Valor de p } \\
\text { *Com } \\
\text { Charlson }\end{array}$ & $\begin{array}{c}\text { RR (IC 95\%) } \\
* \text { Sem Charlson }\end{array}$ & $\begin{array}{c}\text { Valor de p } \\
* * \text { Sem } \\
\text { Charlson }\end{array}$ \\
\hline Idade & NA & NA & $1,028(1,005-1,052)$ & 0,018 \\
\hline ICCharlson & $1,225(1,137-1,320)$ & 0,0001 & NA & NA \\
\hline Doença Renal & NA & NA & $2,971(1,587-5,560)$ & 0,001 \\
\hline Demência & NA & NA & $2,179(1,099-4,318)$ & 0,026 \\
\hline IAM & NA & NA & $1,457(0,881-2,408)$ & 0,142 \\
\hline $\begin{array}{l}\text { Doença Neo- } \\
\text { Hematológica }\end{array}$ & NA & NA & $4,083(0,973-17,140)$ & 0,055 \\
\hline $\begin{array}{l}\text { Insuficiência Tricúspide } \\
\text { Grave }\end{array}$ & $1,63(1,12-2,36)$ & 0,01 & $2,901(1,236-6,809)$ & 0,034 \\
\hline
\end{tabular}

Fonte: criação própria do estudo.

\section{DISCUSSÃO}

A insuficiência cardíaca tem sido alvo de extensas pesquisas com relação à mortalidade e qualidade do atendimento intra-hospitalar. A maior parte das evidências avalia 
os pacientes portadores de IC que internam por descompensação aguda, identificados pelo diagnóstico principal de alta ${ }^{11}$. No entanto, a literatura mostra que a maioria dos pacientes com IC interna por outras causas ${ }^{19,20}$. Um dos poucos estudos que compara essas populações, realizado nos Estados Unidos, demonstrou que o grupo de pacientes portadores de IC que internam por outros diagnósticos não recebe, na mesma proporção que os que internam por IC aguda, os cuidados que sabidamente alteram o prognóstico da doença ${ }^{12}$. Até o presente momento, não há estudos na América Latina que avaliem essa população.

Dentre os resultados encontrados no nosso estudo, é alarmante a taxa de mortalidade. Dos pacientes portadores de IC que internaram no período estudado, 17,4\% foram a óbito ainda na internação. Esse valor é muito superior aos encontrados em outros países inclusive em períodos mais prolongados, como Estados Unidos ${ }^{16}$, Japão ${ }^{21}$ e Canadá ${ }^{22}$, e mesmo aos encontrados em outros estudos brasileiros ${ }^{4,7,8,16}$. Outro fato a ser observado é que a mortalidade intra-hospitalar entre os grupos avaliados (IC aguda versus IC não-aguda) é bastante semelhante $(18,8 \%$ versus $16,8 \%)$, porém um pouco maior no grupo IC aguda, apesar deste possuir uma menor média de ICCharlson. Tal resultado difere do encontrado em estudo israelense ${ }^{14}$ : neste, a mortalidade intra-hospitalar foi maior no grupo de pacientes internados por outras causas que não IC aguda, mas as taxas de mortalidade em 1 ano e em 10 anos foram menores no grupo de pacientes internados por outras causas que não IC aguda. Tal diferença entre os estudos, quanto à mortalidade intra-hospitalar, pode ser reflexo de um déficit de qualidade no atendimento prestado a nossos pacientes internados por IC aguda, mas também pode ser secundária a questões mais complexas e que fogem ao escopo de nosso estudo, tais como a severidade da IC de base de nossos pacientes ou mesmo o seu perfil socioeconômico e cultural.

Merece destaque também o tempo de internação dos nossos pacientes. Alguns estudos já associaram o tempo de permanência hospitalar a maior mortalidade ${ }^{22,23,24}$, o que foi reproduzido na nossa população entre os pacientes que não internaram por IC aguda. A mediana de tempo de internação encontrada foi de 15 dias, enquanto que nos Estados Unidos a duração média foi de 5 dias $^{24}$; e, no Hospital de Clínicas de Porto Alegre, de 11 dias ${ }^{16}$. Comparando-se os dois grupos, aqueles que não internaram por descompensação de IC tiveram maior tempo de internação, o que também já foi demonstrado na literatura 
americana $^{12}$. Uma das possíveis explicações para isso é a maior prevalência de outras comorbidades nos pacientes com IC não-aguda e, por conseguinte, um maior ICCharlson; observa-se, nesse grupo, uma maior prevalência de doença cerebrovascular, infarto agudo do miocárdio, doença vascular periférica, neoplasia e DPOC. Na população americana, observou-se também nos pacientes que não internaram por IC aguda uma maior prevalência de DPOC, asma, doença cerebrovascular e depressão.

A idade em nosso estudo também demonstrou ser um preditor de mortalidade, sendo consistente com achados de outros estudos. No mesmo estudo já citado no Japão, a idade > 70 anos aparece como um marcador de pior prognóstico nos pacientes portadores de $\mathrm{IC}^{21}$. Pocock et al. afirma que idade > 60 anos foi um dos principais preditores para internação por IC, morte de causa cardiovascular ou morte por qualquer causa, com o risco de morte aumentando em 2 vezes a cada 10 anos acima dos $60 \operatorname{anos}^{17}$. Carrasco-Sanchez et al., em seu trabalho com pacientes internados por IC com FE preservada, encontrou nos pacientes $\geq 80$ anos uma mortalidade de $42,7 \%$ e nos pacientes com $<80$ anos uma mortalidade de $26,6 \%$ $(\mathrm{p}<0,001)$, confirmando a idade como um preditor de mortalidade ${ }^{25}$. Outros estudos separando pacientes com IC com $\geq 80$ anos e $<80$ anos também encontraram maior mortalidade nos pacientes mais velhos. ${ }^{26,27}$

Os resultados do nosso estudo levantam a discussão se nosso achado é decorrente de pacientes mais graves (vide ICCharlson na Tabela 1) e complexos, que demandam mais tempo internados; ou também uma pior qualidade assistencial, levando a um maior tempo de permanência do paciente hospitalizado e com isso a um maior número de óbitos. Analisando as características da população do estudo publicado por Rohde et al., encontrou-se um ICCharlson com média de 2,4 na população americana e 2,2 na brasileira ${ }^{16}$, enquanto a média encontrada em nosso estudo foi de 5 , demonstrando claramente um perfil de paciente mais grave.

Há poucos trabalhos que utilizaram o ICCharlson no contexto de insuficiência cardíaca. Em um estudo italiano, o ICCharlson foi usado em pacientes com IC que foram acompanhados por 12 anos e não se comprovou ser um preditor de pior prognóstico nos idosos $(\geq 65 \text { anos })^{28}$. Em contrapartida, Oudejans et al., em uma população holandesa com média de 83 anos e proveniente de casas geriátricas, demonstrou que o ICCharlson foi o 
preditor independente de mortalidade em 3 anos de maior impacto ${ }^{29}$. Da mesma forma, em uma coorte histórica realizada no Canadá com mais de 38700 pacientes internados pela primeira vez por IC aguda, o ICCharlson mostrou ser um bom preditor de mortalidade em 30 dias e em 1 ano.

Não foi avaliado, no nosso estudo, o perfil socioeconômico dos pacientes. Uma revisão de literatura de $2011^{30}$, embora tenha encontrado resultados conflitantes sobre as várias características socioeconômicas e sua associação com prognóstico na IC, chegou às seguintes conclusões: mesmo em uma mesma população, características diferentes como nível educacional e renda são associadas com o desenvolvimento de IC; a junção de vários marcadores de privação social são preditores de maior impacto no desfecho da IC do que os mesmos marcadores de forma isolada; o impacto dos marcadores socioeconômicos varia conforme a faixa etária, sendo os pacientes de meia-idade os mais afetados; e finalmente, as inequidades entre o sistema de saúde universal e o privado sugerem que diferenças no tratamento ou cuidado de especialistas não são os únicos responsáveis por desfechos diferentes. Como hospital inteiramente público, em um País que ainda enfrenta grande desigualdade social, infere-se que o nível socioeconômico dos pacientes atendidos no HNSC esteja aquém do encontrado nos EUA e Europa. Estudos adicionais devem ser realizados para aferir a real repercussão de tais fatores no desfecho da IC.

Ao analisar as tabelas 4 e 5 do nosso estudo constatou-se que o grupo de pacientes que tinha IC, mas que não internou por IC aguda, apresentou preditores de mortalidade intrahospitalar muito semelhantes ao grupo de pacientes que internou por IC aguda. Assim, observamos a necessidade de se pesquisar mais significativamente essa população aparentemente heterogênea, haja vista a semelhança de indicadores hospitalares e preditores de mortalidade.

\section{CONCLUSÃO}

Nosso trabalho é um dos primeiros da América Latina a confirmar que o perfil de pacientes com IC é diferente daqueles estudados na América do Norte e Europa, 
demonstrando uma população idosa, com mais comorbidades e maior tempo de internação, o que retrata a realidade de um hospital terciário brasileiro. Trata-se também de um dos poucos estudos existentes a ressaltar a importância do ICCharlson em predizer mortalidade nesta população com IC, além de um dos poucos a analisar as características de uma população portadora de IC que interna por outros motivos que não a IC aguda.

Apesar de termos pacientes com características diferentes da literatura internacional, constatamos que a maioria dos preditores de mortalidade intra-hospitalar encontrados na nossa amostra é muito semelhante aos dos estudos já publicados. Além disso, apesar de termos estudado duas populações diferentes (uma de pacientes que não internaram por IC aguda e uma que internou por essa causa) os preditores de mortalidade intra-hospitalar encontrados nos dois grupos foram muito semelhantes.

Tendo em vista as limitações inerentes ao nosso estudo como ser observacional, retrospectivo e com análise limitada a prontuários, necessita-se de mais pesquisas na América Latina para conhecer-se mais adequadamente a realidade dos pacientes com IC no nosso meio.

\section{Evaluation of intra-hospital mortality predictors in Heart Failure patients in a tertiary hospital in Brazil}

\section{Abstract}

Introduction: Heart failure $(H F)$ is the major cardiovascular cause of hospital morbidity. In spite of its relevance, studies on intra-hospital mortality predictors in patients suffering from HF are scarce in Latin America.

Objective: To identify the intra-hospital mortality predictors in patients who are admitted due to acute HF or who suffer from HF and are admitted for other reasons in Hospital Nossa Senhora da Conceição (HNSC), Porto Alegre, Brazil. 
Methodology: Historic cohort of patients who were hospitalized with a diagnosis of HF according to the Charlson comorbidity index (CCI) in 2009 and 2010.

Results: A total of 2,056 patients (51\% males and 49\% females), admitted between January 1, 2009 and December 31, 2010 was evaluated. Their average age was 71 years; the average CCI was 5 and acute HF was responsible for $28.7 \%$ of the admissions. We have found in our sample 361 (17.4\%) deaths during the admission, being $18.8 \%$ in the group of patients who was admitted for acute HF and $16.8 \%$ in the group with HF who was admitted for other reasons. The main intra-hospital mortality predictors in the group who was not admitted for acute HF were: age $(R R=1.023$; CI 95\% 1.011-1.036); hospital stay $(R R=1.009 ;$ CI 95\% 1.005 - 1.013); CCI $(R R=1.144$; CI 95\% $1.098-$ 1.193); kidney disease ( $R R=2.49$; CI 95\% 1.71 - 3.63); dementia $(R R=1.94$; CI 95\% 1.35 - 2.77) and hematologic disease ( $R R=3,07$; IC95\% 1,51-6,72). In the group who was admitted for acute $H F$, the main independent mortality predictors were: age ( $R R=1.028$; CI 95\% 1.005-1.052); CCI $(R R=1.225$; $C I$ $95 \% 1.137$ - 1.320); kidney disease (RR=2.97; CI 95\% 1.587 - 5,560); dementia $(R R=2.179$ CI 95\% 1.099-4.318); and acute myocardial infarction $(R R=1.85 ; C I 95 \% 1.26-2.71)$.

Conclusions: Our study showed a profile of patients with HF different from those studied in North America and Europe. It is one of the few existing studies highlighting the importance of the CCI to predict mortality in patients with $\mathrm{HF}$ and to analyze the characteristics of population of HF patients who is admitted for reasons other than acute HF. Besides, we have found that, in spite of having patients with characteristics that are different from the international literature, the majority of the intra-hospital mortality predictors found in our study is quite similar to those from studies already published, as well as being very similar between both groups analyzed by our study.

Keywords: Intra-hospital mortality, heart failure, cardiovascular disease 


\section{Referências}

1- MCDONAGH TA, BLUE L, CLARK AL, DAHLSTRÖM U, EKMAN I, LAINSCAK M, MCDONALD K, RYDER M, STRÖMBERG A, JAARSMA T; European Society of Cardiology Heart Failure Association Committee on Patient Care; European Society of Cardiology Heart Failure Association Standards for delivering heart failure care; Eur J Heart Fail; 2011 Mar; 13(3): 235-41.

2- COWIE MR, WOOD DA, COATS AJ, THOMPSON SG, POOLE-WILSON PA, SURESH V, SUTTON GC. Incidence and aetiology of heart failure; a population-based study. Eur Heart J 1999;20:421-428.

3- American Heart Association Statistics Committee and Stroke Statistics Subcommittee. Heart disease and stroke statistics--2014 update: a report from the American Heart Association. Circulation; 2014 Jan 21;129(3): e28-e292.

4- BARRETTO AC, DEL CARLO CH, CARDOSO JN, MORGADO PC, MUNHOZ RT, EID MO, OLIVEIRA MT JR, SCIPIONI AR, RAMIRES JA; Hospital readmissions and death from Heart Failure - rates still alarming; Arq Bras Cardiol.; 2008 Nov; 91(5): 335-41.

5- GHEORGHIADE M, BRAUNWALD E. Hospitalizations for heart failure in the United States-a sign of hope. JAMA 2011;306:1705-6.

6- BOCCHI EA. Heart failure in South America. Curr Cardiol Rev. 2013;9(2):147-56.

7- Ministério da Saúde. Datasus: mortalidade - 1996 a 2012, pela CID-10 - Brasil [Internet]. Brasília (DF); 2008. [citado em 2014 dez 03]. Disponível em: http://tabnet.datasus.gov.br/cgi/deftohtm.exe?sim/cnv/obt10uf.def

8- DENILSON CAMPOS DE ALBUQUERQUE, JOÃO DAVID DE SOUZA, NETO, FERNANDO BACAL, LUIZ EDUARDO PAIM ROHDE, SABRINA BERNARDEZPEREIRA, OTAVIO BERWANGER, DIRCEU RODRIGUES ALMEIDA, and Investigadores Estudo BREATHE. I Brazilian Registry of Heart Failure - Clinical Aspects, Care Quality and Hospitalization Outcomes. Arq Bras Cardiol. 2015 Jun; 104(6): 433-442.

9- SAUL BLECKER, SUNIL K. AGARWAL, PATRICIA P. CHANG, WAYNE D. ROSAMOND, DONALD E. CASEY, ANNA KUCHARSKA-NEWTON, MARTHA J. RADFORD, JOSEF CORESH, STUART KATZ. Quality of Care for Heart Failure Patients Hospitalized for Any Cause. J Am Coll Cardiol. 2014 January 21; 63(2): 123-130.

10- WRITING COMMITTEE M, YANCY CW, JESSUP M, ET AL. 2013 ACCF/AHA guideline for the management of heart failure: a report of the American College of Cardiology Foundation/American Heart Association Task Force on practice guidelines. Circulation 2013;128:e240-327.

11- BONOW, R. O., GANIATS, T. G., BEAM, C. T., BLAKE, K., CASEY, D. E., GOODLIN, S. J.,GRADY, K. L., ET AL; ACCF/AHA/AMA-PCPI 2011 performance 
measures for adults with heart failure: a report of the American College of Cardiology Foundation/American Heart Association Task Force on Performance Measures and the American Medical Association-Physician Consortium for Performance Improvement; Circulation; 2012 May 15; 125(19): 2382-401.

12- BLECKER S, AGARWAL S, CHANG P, ROSAMOND W, CASEY D, KUCHARSKANEWTON A, RADFORD M, CORESH J, KATZ S. Quality of care for heart failure patients hospitalized for any cause. Journal of the American College of Cardiology. 2014; 63:123130 .

13- AKSHAY S. DESAI; BRIAN CLAGGETT; MARC A. PFEFFER; NATALIE BELLO; PETER V. FINN; CHRISTOPHER B. GRANGER; JOHN J.V. MCMURRAY; STUART POCOCK; KARL SWEDBERG; SALIM YUSUF; SCOTT D. SOLOMON. Influence of Hospitalization for Cardiovascular Versus Noncardiovascular Reasons on Subsequent Mortality in Patients With Chronic Heart Failure Across the Spectrum of Ejection Fraction. Circ Heart Fail. 2014;7:895-902.

14- EREZ A, KLEMPFNER R, GOLDENBERG I, ELIS A. Short and long term survival following hospitalization with a primary versus non-primary diagnosis of acute heart failure. Eur J Intern Med. 2015 Jul;26(6):420-4.

15- SULO G, IGLAND J, VOLLSET SE, NYGÅRD O, EBBING M, SULO E, EGELAND GM, TELL GS. Heart Failure Complicating Acute Myocardial Infarction; Burden and Timing of Occurrence: A Nation-wide Analysis Including 86.771 Patients From the Cardiovascular Disease in Norway (CVDNOR) Project. J Am Heart Assoc. 2016 Jan 7;5(1).

16- ROHDE LE, CLAUSELL N, RIBEIRO JP, GOLDRAICH L, NETTO R, WILLIAM DEC G, DISALVO TG, POLANCZYK CA.; Health outcomes in decompensated congestive heart failure: a comparison of tertiary hospitals in Brazil and United States; Int J Cardiol; 2005 Jun 22; 102(1): 71-7.

17- POCOCK SJ, WANG D, PFEFFER MA, YUSUF S, MCMURRAY JJ, SWEDBERG KB, OSTERGREN J, MICHELSON EL, PIEPER KS, GRANGER CB; Predictors of mortality and morbidity in patients with chronic heart failure; Eur Heart J.; 2006 Jan; 27(1): $65-75$.

18- CHARLSON ME, POMPEI P, ALES KL, MACKENZIE CR; A new method of classifying prognostic comorbidity in longitudinal studies: development and validation; Journal of chronic diseases [Internet]; 1987 Jan [cited 2011 Jul 19]; 40(5): 373-83.

19- DUNLAY SM, REDFIELD MM, WESTON SA, ET AL. Hospitalizations after heart failure diagnosis a

community perspective. J Am Coll Cardiol. 2009; 54:1695-1702. [PubMed: 19850209] 
20- BLECKER S, PAUL M, TAKSLER G, OGEDEGBE G, KATZ S. Heart failure associated hospitalizations in the United States. J Am Coll Cardiol. 2013; 61:1259-1267. [PubMed: 23500328].

21- KAWASHIRO N, KASANUKI H, OGAWA H, MATSUDA N, HAGIWARA N; Clinical characteristics and outcome of hospitalized patients with congestive heart failure: results of the HIJC-HF registry; Circ J; 2008 Dec; 72(12): 2015-20.

22- MUZZARELLI S, LEIBUNDGUT G, MAEDER M, HICKLI H, HANDSCHIN R, GURMANN M, JEKER U, BUSER P, PFISTERER M, BRUNNER-LA ROCCA HP. Predictors of early readmission or death in elderly patients with heart failure. American Heart Journal. 2010; 160 (2), pp. 308-314.

23. REYNOLDS K, BUTLER MG, KIMES TM, ET AL. Relation of Acute Heart Failure Hospital Length of Stay to Subsequent Readmission and All-Cause Mortality. Am J Cardiol. 2015; 116:400-405.

24- PETERSON PN, RUMSFELD JS, LIANG L, ALBERT NM, HERNANDEZ AF, PETERSON ED, FONAROW GC, MASOUDI FA; American Heart Association Get With the Guidelines-Heart Failure Program; A Validated Risk Score for In-Hospital Mortality in Patients With Heart Failure From the American Heart Association Get With the Guidelines Program. ; 2010 Jan; 3(1): 25-32.

25- CARRASCO SANCHEZ FJ, RUBIO MIP, MORENO JMG, GARCIA IV, SANABRIA JÁ, LLAVE EP; Variables predictoras de mortalidad en pacientes ancianos hospitalizados por insuficiência cardíaca com fracción de eyección preservada; MedClin (Barc); 2013; 141(10): 423-429.

26- HAMAGUCHI S, KINUGAWA S, GOTO D, TSUCHIHASHI-MAKAYA M, YOKOTA T, YAMADA S, YOKOSHIKI H, TAKESHITA A, TSUTSUIH. Predictors of Long-Term Adverse Outcomes in Elderly Patients Over 80 Years Hospitalized With Heart Failure - A Report From the Japanese Cardiac Registry of Heart Failure in Cardiology (JCARE-CARD); Circulation Journal; 2011 Oct; Vol.75(10), pp.2403-2410.

27- MAHJOUB H, RUSINARU D, SOULIÈRE V, DURIER C, PELTIER M, TRIBOUILLOY; Long C-term survival in patients older than 80 years hospitalised for heart failure. A 5-year prospective study; Eur J Heart Fail; 2008; 10: 78-84.

28- TESTA G, CACCIATORE F, GALIZIA G, DELlA-MORTE D, MAZZELLA F, RUSSO S, FERRARA N, RENGO F, ABETE P; Charlson Comorbidity Index does not predict long-term mortality in elderly subjects with chronic heart failure; Age Ageing.; 2009 Nov; 38(6): 734-40.

29- OUDEJANS I, MOSTERD A, ZUITHOFF NP, HOES AW. Comorbidity Drives Mortality in Newly Diagnosed Heart Failure: A Study Among Geriatric Outpatients; J Card Fail; 2012 Jan; 18(1): 47-52. 
30- HAWKINS, N. M., JHUND, P. S., MCMURRAY, J. J.V. AND CAPEWELL, S. (2012), Heart failure and socioeconomic status: accumulating evidence of inequality. European Journal of Heart Failure, 14: 138-146. doi: 10.1093/eurjhf/hfr168 